\title{
New Simpson Type Integral Inequalities for $s$-Convex Functions and Their Applications
}

\author{
Artion Kashuri, ${ }^{1}$ Pshtiwan Othman Mohammed $\mathbb{D}^{2}{ }^{2}$ Thabet Abdeljawad $\left(\mathbb{D},{ }^{3,4,5}\right.$ \\ Faraidun Hamasalh, ${ }^{2}$ and Yuming Chu $\mathbb{D}^{6,7}$ \\ ${ }^{1}$ Department of Mathematics, Faculty of Technical Science, University Ismail Qemali, Vlora 9401, Albania \\ ${ }^{2}$ Department of Mathematics, College of Education, University of Sulaimani, Sulaimani 46001, Kurdistan Region, Iraq \\ ${ }^{3}$ Department of Mathematics and General Sciences, Prince Sultan University, P.O. Box 66833, Riyadh 11586, Saudi Arabia \\ ${ }^{4}$ Department of Medical Research, China Medical University, Taichung 40402, Taiwan \\ ${ }^{5}$ Department of Computer Science and Information Engineering, Asia University, Taichung 41354, Taiwan \\ ${ }^{6}$ Department of Mathematics, Huzhou University, Huzhou 313000, China \\ ${ }^{7}$ Hunan Provincial Key Laboratory of Mathematical Modeling and Analysis in Engineering, \\ Changsha University of Science \& Technology, Changsha 410114, China
}

Correspondence should be addressed to Thabet Abdeljawad; tabdeljawad@psu.edu.sa and Yuming Chu; chuyuming@zjhu.edu.cn

Received 16 September 2020; Revised 6 October 2020; Accepted 10 October 2020; Published 26 October 2020

Academic Editor: Imtiaz Ahmad

Copyright $(92020$ Artion Kashuri et al. This is an open access article distributed under the Creative Commons Attribution License, which permits unrestricted use, distribution, and reproduction in any medium, provided the original work is properly cited.

First, we consider a new Simpson's identity. This identity investigates our main results that consist of some integral inequalities of Simpson's type for the $s$-convex functions. From our main results, we obtain some special cases which are discussed in detail. Finally, some applications on the Bessel functions, special means of distinct positive real numbers, and error estimation about Simpson quadrature formula are presented to support our theoretical results.

\section{Introduction and Preliminaries}

Integral inequalities are generally applicable in many branches of mathematics such as mathematical analysis, fractional calculus, discrete fractional calculus, and abstract spaces; for an overview, the reader should see the literature on integral inequalities, e.g., [1-11] and the references therein.

Nowadays, the study of convexity is considered as an original icon in the investigation of theoretical behavior of mathematical inequalities (e.g., [12-15]). Recently, several works on integral inequalities for convex functions were conducted. In particular, much attention has been given to the theoretical studies of inequalities on different types of convex functions such as $s$-geometrically convex functions [16], GA-convex functions [17], quasiconvex function [18], MT-convex function $[19,20],(\alpha, m)$-convex functions [21], $F$-convex functions [22], $\lambda_{\psi}$-convex functions [23], and a new class of convex functions [24], and many other types can be found in [25].
Now we recall some useful definitions of convex functions that are essential for our present investigation.

Definition 1 (see [25]). A function $f: \mathscr{T} \subseteq \mathbb{R} \longrightarrow \mathbb{R}$ is said to be convex, if

$$
f\left(\nu \omega_{1}+(1-v) \omega_{2}\right) \leq \nu f\left(\omega_{1}\right)+(1-v) f\left(\omega_{2}\right)
$$

holds for all $\omega_{1}, \omega_{2} \in \mathscr{T}$ and $v \in[0,1]$. Likewise, $f$ is concave if $(-f)$ is convex.

Definition 2 (see [26]). Let $s \in(0,1]$ be fixed. A function $f: \mathscr{T} \subseteq \mathbb{R} \longrightarrow \mathbb{R}$ is said to be $s$-convex (in the second sense), if

$$
f\left(\nu \omega_{1}+(1-v) \omega_{2}\right) \leq \nu^{s} f\left(\omega_{1}\right)+(1-\nu)^{s} f\left(\omega_{2}\right)
$$

holds for all $\omega_{1}, \omega_{2} \in \mathscr{T}$ and $\nu \in[0,1]$.

Recently, many well-known inequalities haven been established for the convex function and its types such as 
Hermite-Hadamard type inequalities [27, 28], Hermite-Hadamard-Fejer type inequalities [29], Ostrowski type inequalities [30], Hardy type inequalities [31], Olsen type inequalities [32], Gagliardo-Nirenberg type inequalities [33], Opial type inequalities [34, 35], and Rozanova type inequalities [36]. However, our focus in this paper is on another type of inequality, namely, the Simpson's inequality, which is obtained by Dragomir et al. [37], and their main result is as follows.

Theorem 1 (see [37]). Assume that $f:\left[\omega_{1}, \omega_{2}\right] \longrightarrow \mathbb{R}$ is a four times continuous and differentiable function on $\left(\omega_{1}, \omega_{2}\right)$ such that $\left\|f^{(4)}\right\|_{\infty}:=\sup _{x \in\left(\omega_{1}, \omega_{2}\right)}\left|f^{(4)}(x)\right|<+\infty$ with $\omega_{1}<\omega_{2}$. Then, the following integral inequality holds:

$$
\begin{aligned}
& \mid \frac{1}{6}\left[f\left(\omega_{1}\right)+4 f\left(\frac{\omega_{1}+\omega_{2}}{2}\right)+f\left(\omega_{2}\right)\right] \\
& \quad-\frac{1}{\omega_{2}-\omega_{1}} \int_{\omega_{1}}^{\omega_{2}} f(x) \mathrm{d} x \mid \leq \frac{1}{2880}\left(\omega_{2}-\omega_{1}\right)^{4}\left\|f^{(4)}\right\|_{\infty} .
\end{aligned}
$$

Just like the aforementioned inequalities, several integral inequalities related to the Simpson's integral inequality (3) has been found for the convex functions (see, e.g., [38-45]).
In view of the above indices and literatures, we will prove a new Simpson's integral identity. From this, then we will obtain several Simpson's integral inequalities and some special cases.

The rest of this article is organized in the following way: Section 2 contains the main results of the article which consists of proving several Simpson's integral inequalities and some related results. In Section 3, we give some applications of the Bessel functions, special means, and error estimation about the Simpson quadrature formula to support the main results. Section 4 is for the conclusions.

\section{Main Results}

We need the following Lemma in order to establish our main results.

Lemma 1. Let $f: \mathscr{T} \subseteq \mathbb{R} \longrightarrow \mathbb{R}$ be an $\mathscr{L}^{1}$ function on $\mathscr{T}$ such that $\omega_{1}, \omega_{2} \in \mathscr{T}$ with $\omega_{1}<\omega_{2}$. Also, let $f^{\prime} \in \mathscr{L}^{1}$, and then for any positive integer number $n$, we have

$$
\begin{aligned}
T_{n}\left(f ; \omega_{1}, \omega_{2}\right): & =\sum_{k=0}^{n-1} \frac{1}{6 n}\left[f\left(\frac{(n-k) \omega_{1}+k \omega_{2}}{n}\right)+4 f\left(\frac{(2(n-k)-1) \omega_{1}+(2 k+1) \omega_{2}}{2 n}\right)\right. \\
& \left.+f\left(\frac{(n-k-1) \omega_{1}+(k+1) \omega_{2}}{n}\right)\right]-\frac{1}{\omega_{2}-\omega_{1}} \int_{\omega_{1}}^{\omega_{2}} f(x) \mathrm{d} x \\
= & \left(\frac{\omega_{2}-\omega_{1}}{n^{2}}\right) \sum_{k=0}^{n-1} \int_{0}^{1} \Phi(\nu) f^{\prime}\left((1-v) \frac{(n-k) \omega_{1}+k \omega_{2}}{n}+v \frac{(n-k-1) \omega_{1}+(k+1) \omega_{2}}{n}\right) \mathrm{d} \nu
\end{aligned}
$$

where

$$
\Phi(v)= \begin{cases}v-\frac{1}{6}, & v \in\left[0, \frac{1}{2}\right), \\ v-\frac{5}{6}, & v \in\left[\frac{1}{2}, 1\right] .\end{cases}
$$

$$
\begin{aligned}
& \left(\frac{\omega_{2}-\omega_{1}}{n^{2}}\right) \int_{0}^{1} \Phi(\nu) f^{\prime}\left((1-v) \frac{(n-k) \omega_{1}+k \omega_{2}}{n}+v \frac{(n-k-1) \omega_{1}+(k+1) \omega_{2}}{n}\right) \mathrm{d} \nu \\
& =\left(\frac{\omega_{2}-\omega_{1}}{n^{2}}\right)\left\{\int_{0}^{(1 / 2)}\left(\nu-\frac{1}{6}\right) f^{\prime}\left((1-v) \frac{(n-k) \omega_{1}+k \omega_{2}}{n}+v \frac{(n-k-1) \omega_{1}+(k+1) \omega_{2}}{n}\right) \mathrm{d} \nu\right. \\
& \left.\quad+\int_{(1 / 2)}^{1}\left(v-\frac{5}{6}\right) f^{\prime}\left((1-v) \frac{(n-k) \omega_{1}+k \omega_{2}}{n}+v \frac{(n-k-1) \omega_{1}+(k+1) \omega_{2}}{n}\right) \mathrm{d} \nu\right\} .
\end{aligned}
$$


By applying the integration by parts to the right side of (6), we have

$$
\begin{aligned}
& \int_{0}^{(1 / 2)}\left(\nu-\frac{1}{6}\right) f^{\prime}\left((1-v) \frac{(n-k) \omega_{1}+k \omega_{2}}{n}+v \frac{(n-k-1) \omega_{1}+(k+1) \omega_{2}}{n}\right) \mathrm{d} \nu \\
& =\left(\frac{n}{\omega_{2}-\omega_{1}}\right)\left[\left.\left(\nu-\frac{1}{6}\right) f\left((1-v) \frac{(n-k) \omega_{1}+k \omega_{2}}{n}+v \frac{(n-k-1) \omega_{1}+(k+1) \omega_{2}}{n}\right)\right|_{t=0} ^{(1 / 2)}\right. \\
& \left.\quad-\int_{0}^{(1 / 2)} f\left((1-v) \frac{(n-k) \omega_{1}+k \omega_{2}}{n}+v \frac{(n-k-1) \omega_{1}+(k+1) \omega_{2}}{n}\right) \mathrm{d} v\right] \\
& =\left(\frac{n}{\omega_{2}-\omega_{1}}\right)\left[\frac{1}{3} f\left(\frac{(2(n-k)-1) \omega_{1}+(2 k+1) \omega_{2}}{2 n}\right)+\frac{1}{6} f\left(\frac{(n-k) \omega_{1}+k \omega_{2}}{n}\right)\right. \\
& \left.\quad-\int_{0}^{(1 / 2)} f\left((1-v) \frac{(n-k) \omega_{1}+k \omega_{2}}{n}+v \frac{(n-k-1) \omega_{1}+(k+1) \omega_{2}}{n}\right) \mathrm{d} v\right]
\end{aligned}
$$

and similarly,

$$
\begin{aligned}
& \int_{(1 / 2)}^{1}\left(\nu-\frac{5}{6}\right) f^{\prime}\left((1-v) \frac{(n-k) \omega_{1}+k \omega_{2}}{n}+v \frac{(n-k-1) \omega_{1}+(k+1) \omega_{2}}{n}\right) d v \\
& =\left(\frac{n}{\omega_{2}-\omega_{1}}\right)\left[\frac{1}{3} f\left(\frac{(2(n-k)-1) \omega_{1}+(2 k+1) \omega_{2}}{2 n}\right)+\frac{1}{6} f\left(\frac{(n-k-1) \omega_{1}+(k+1) \omega_{2}}{n}\right)\right. \\
& \left.\quad-\int_{(1 / 2)}^{1} f\left((1-v) \frac{(n-k) \omega_{1}+k \omega_{2}}{n}+v \frac{(n-k-1) \omega_{1}+(k+1) \omega_{2}}{n}\right) d v\right],
\end{aligned}
$$

for all $k=0,1,2, \ldots, n-1$. Then, substituting (7) and (8) in the equality (6), we get

$$
\begin{aligned}
& \left(\frac{\omega_{2}-\omega_{1}}{n^{2}}\right) \int_{0}^{1} \Phi(\nu) f^{\prime}\left((1-v) \frac{(n-k) \omega_{1}+k \omega_{2}}{n}+v \frac{(n-k-1) \omega_{1}+(k+1) \omega_{2}}{n}\right) \mathrm{d} \nu \\
& =\frac{1}{6 n}\left[f\left(\frac{(n-k) \omega_{1}+k \omega_{2}}{n}\right)+4 f\left(\frac{(2(n-k)-1)_{1}+(2 k+1) \omega_{2}}{2 n}\right)\right. \\
& \left.\quad+f\left(\frac{(n-k-1) \omega_{1}+(k+1) \omega_{2}}{n}\right)\right]-\frac{1}{\omega_{2}-\omega_{1}} \int_{\left((n-k) \omega_{1}+k \omega_{2} / n\right)}^{\left((n-k-1) \omega_{1}+(k+1) \omega_{2} / n\right)} f(x) \mathrm{d} x .
\end{aligned}
$$

By summing over $k$ from 0 to $n-1$, we can deduce the desired identity (4).
Remark 1. Taking $n=1$ in Lemma 1 , we have the following Simpson's rule (see [43]):

$$
\frac{1}{6}\left[f\left(\omega_{1}\right)+4 f\left(\frac{\omega_{1}+\omega_{2}}{2}\right)+f\left(\omega_{2}\right)\right]-\frac{1}{\omega_{2}-\omega_{1}} \int_{\omega_{1}}^{\omega_{2}} f(x) \mathrm{d} x=\left(\omega_{2}-\omega_{1}\right) \int_{0}^{1} \Phi(\nu) f^{\prime}\left(\nu \omega_{2}+(1-\nu) \omega_{1}\right) \mathrm{d} \nu
$$


where $\omega(\nu)$ is defined by (5).

Theorem 2. Let $f: \mathscr{T} \subseteq[0,+\infty) \longrightarrow \mathbb{R}$ be an $\mathscr{L}^{1}$ function on $\mathscr{T}$ such that $\omega_{1}, \omega_{2} \in \mathscr{T}$ with $\omega_{1}<\omega_{2}$ and $s \in(0,1]$ be fixed.
Also, let $f^{\prime} \in \mathscr{L}^{1}$ and $\left|f^{\prime}\right|$ be an s-convex function on $\left[\omega_{1}, \omega_{2}\right]$, and then for any positive integer number $n$, we have

$$
\left|T_{n}\left(f ; \omega_{1}, \omega_{2}\right)\right| \leq\left(\frac{\omega_{2}-\omega_{1}}{n^{2}}\right) \sum_{k=0}^{n-1}\left[\left(A_{1}(s)+A_{3}(s)\right)\left|f^{\prime}\left(\mathbf{e}_{n, k}\right)\right|+\left(A_{2}(s)+A_{4}(s)\right)\left|f^{\prime}\left(\mathbf{e}_{n, k+1}\right)\right|\right],
$$

where

$$
\begin{aligned}
A_{1}(s):= & \int_{0}^{(1 / 2)}\left|v-\frac{1}{6}\right|(1-v)^{s} \mathrm{~d} v=\frac{1}{6}\left[\frac{1-(5 / 6)^{s+1}}{s+1}\right]-\frac{1-(5 / 6)^{s+1}}{s+1}+\frac{1-(5 / 6)^{s+2}}{s+2} \\
& +\frac{(5 / 6)^{s+1}-(1 / 2)^{s+1}}{s+1}-\frac{(5 / 6)^{s+2}-(1 / 2)^{s+2}}{s+2}-\frac{1}{6}\left[\frac{(5 / 6)^{s+1}-(1 / 2)^{s+1}}{s+1}\right], \\
A_{2}(s):= & \int_{0}^{(1 / 2)}\left|v-\frac{1}{6}\right| \nu^{s} \mathrm{~d} v=\frac{1}{6(s+1)}\left(\frac{2-3^{s+1}}{6^{s+1}}\right)+\frac{1}{s+2}\left(\frac{3^{s+2}-2}{6^{s+2}}\right), \\
A_{3}(s):= & \int_{(1 / 2)}^{1}\left|\nu-\frac{5}{6}\right|(1-v)^{s} \mathrm{~d} v=\frac{5}{6}\left[\frac{(1 / 2)^{s+1}-(1 / 6)^{s+1}}{s+1}\right]-\frac{(1 / 2)^{s+1}-(1 / 6)^{s+1}}{s+1}+\frac{(1 / 2)^{s+2}-(1 / 6)^{s+2}}{s+2} \\
& +\frac{1}{6^{s+1}(s+1)}-\frac{1}{6^{s+2}(s+2)}-\frac{5}{6^{s+2}(s+1)}, \\
A_{4}(s):= & \int_{(1 / 2)}^{1}\left|\nu-\frac{5}{6}\right| v^{s} \mathrm{~d} v=\frac{5}{6}\left[\frac{(5 / 6)^{s+1}-(1 / 2)^{s+1}}{s+1}\right]-\frac{(5 / 6)^{s+2}-(1 / 2)^{s+2}}{s+2}+\frac{1-(5 / 6)^{s+2}}{s+2}-\frac{5}{6}\left[\frac{1-(5 / 6)^{s+1}}{s+1}\right], \\
e_{n, k}:= & \frac{(n-k) \omega_{1}+k \omega_{2}}{n} .
\end{aligned}
$$

Proof. By using Lemma 1, s-convexity of $\left|f^{\prime}\right|$ and properties of modulus, we can deduce

$$
\begin{aligned}
\left|T_{n}\left(f ; \omega_{1}, \omega_{2}\right)\right| & \leq\left(\frac{\omega_{2}-\omega_{1}}{n^{2}}\right) \sum_{k=0}^{n-1} \int_{0}^{1}|\Phi(\nu)|\left|f^{\prime}\left((1-v) \mathbf{e}_{n, k}+\nu \mathbf{e}_{n, k+1}\right)\right| \mathrm{d} v \\
& \leq\left(\frac{\omega_{2}-\omega_{1}}{n^{2}}\right) \sum_{k=0}^{n-1} \int_{0}^{1}|\Phi(\nu)|\left[(1-v)^{s}\left|f^{\prime}\left(\mathbf{e}_{n, k}\right)\right|+\nu^{s}\left|f^{\prime}\left(\mathbf{e}_{n, k+1}\right)\right|\right] \mathrm{d} v \\
& =\left(\frac{\omega_{2}-\omega_{1}}{n^{2}}\right) \sum_{k=0}^{n-1}\left[\left(A_{1}(s)+A_{3}(s)\right)\left|f^{\prime}\left(\mathbf{e}_{n, k}\right)\right|+\left(A_{2}(s)+A_{4}(s)\right)\left|f^{\prime}\left(\mathbf{e}_{n, k+1}\right)\right|\right],
\end{aligned}
$$

which completes the proof.

$$
\left|T_{n}\left(f ; \omega_{1}, \omega_{2}\right)\right| \leq\left(\frac{\omega_{2}-\omega_{1}}{n^{2}}\right) \sum_{k=0}^{n-1}\left[\left(A_{1}+A_{3}\right)\left|f^{\prime}\left(\mathbf{e}_{n, k}\right)\right|\right.
$$

Corollary 1. Choosing $s=1$ in Theorem 2, we get the following inequality for the convex function:

$$
\left.+\left(A_{2}+A_{4}\right)\left|f^{\prime}\left(\mathbf{e}_{n, k+1}\right)\right|\right]
$$


where

$$
\begin{aligned}
A_{1}:= & \int_{0}^{(1 / 2)}\left|\nu-\frac{1}{6}\right|(1-v) \mathrm{d} v=\frac{1}{6}\left[\frac{1-(5 / 6)^{2}}{2}\right]-\frac{1-(5 / 6)^{2}}{2}+\frac{1-(5 / 6)^{3}}{3} \\
& +\frac{(5 / 6)^{2}-(1 / 2)^{2}}{2}-\frac{(5 / 6)^{3}-(1 / 2)^{3}}{3}-\frac{1}{6}\left[\frac{\left.(5 / 6)^{2}-(1 / 2)^{2}\right]}{2}\right] \\
A_{2}:= & \int_{0}^{(1 / 2)}\left|\nu-\frac{1}{6}\right| \nu \mathrm{d} v=\frac{29}{1296} \\
A_{3}:= & \int_{(1 / 2)}^{1}\left|\nu-\frac{5}{6}\right|(1-v) \mathrm{d} v=\frac{5}{6}\left[\frac{(1 / 2)^{2}-(1 / 6)^{2}}{2}\right]-\frac{(1 / 2)^{2}-(1 / 6)^{2}}{2}+\frac{(1 / 2)^{3}-(1 / 6)^{3}}{3}+\frac{1}{1296} \\
A_{4}:= & \int_{(1 / 2)}^{1}\left|\nu-\frac{5}{6}\right| \nu \mathrm{d} v=\frac{5}{6}\left[\frac{(5 / 6)^{2}-(1 / 2)^{2}}{2}\right]-\frac{(5 / 6)^{3}-(1 / 2)^{3}}{3}+\frac{1-(5 / 6)^{3}}{3}-\frac{5}{6}\left[\frac{1-(5 / 6)^{2}}{2}\right]
\end{aligned}
$$

Corollary 2. Choosing $n=1$ in Theorem 2, we get

$$
\begin{aligned}
& \left|\frac{1}{6}\left[f\left(\omega_{1}\right)+4 f\left(\frac{\omega_{1}+\omega_{2}}{2}\right)+f\left(\omega_{2}\right)\right]-\frac{1}{\omega_{2}-\omega_{1}} \int_{\omega_{1}}^{\omega_{2}} f(x) \mathrm{d} x\right| \leq\left(\omega_{2}-\omega_{1}\right) \\
& \quad \times\left[\left(A_{1}(s)+A_{3}(s)\right)\left|f^{\prime}\left(\omega_{1}\right)\right|+\left(A_{2}(s)+A_{4}(s)\right)\left|f^{\prime}\left(\omega_{2}\right)\right|\right] .
\end{aligned}
$$

Theorem 3. Let $f: \mathscr{T} \subseteq[0,+\infty) \longrightarrow \mathbb{R}$ be an $\mathscr{L}^{1}$ function on $\mathscr{T}$ such that $\omega_{1}, \omega_{2} \in \mathscr{T}$ with $\omega_{1}<\omega_{2}$ and $s \in(0,1]$ be fixed.
Also, let $f^{\prime} \in \mathscr{L}^{1}$ and $\left|f^{\prime}\right|^{q}, q>1$ be an s-convex function on $\left[\omega_{1}, \omega_{2}\right]$, and then for any positive integer number $n$, we have

$$
\left|T_{n}\left(f ; \omega_{1}, \omega_{2}\right)\right| \leq\left(\frac{\omega_{2}-\omega_{1}}{n^{2}}\right)\left[\frac{2}{p+1}\left(\frac{2^{p+1}+1}{6^{p+1}}\right)\right]^{(1 / p)}\left(\frac{1}{s+1}\right)^{(1 / q)} \sum_{k=0}^{n-1}\left[\left|f^{\prime}\left(\mathbf{e}_{n, k}\right)\right|^{q}+\left|f^{\prime}\left(\mathbf{e}_{n, k+1}\right)\right|^{q}\right]^{(1 / q)},
$$

where $\mathbf{e}_{n, k}$ is as defined in Theorem 2 and $(1 / p)+(1 / q)=1$.

Proof. By making use of Lemma 1, Hölder's inequality, $s$-convexity of $\left|f^{\prime}\right|^{q}$, and properties of modulus, we have

$$
\begin{aligned}
\left|T_{n}\left(f ; \omega_{1}, \omega_{2}\right)\right| & \leq\left(\frac{\omega_{2}-\omega_{1}}{n^{2}}\right) \sum_{k=0}^{n-1} \int_{0}^{1}|\emptyset(\nu)|\left|f^{\prime}\left((1-v) \mathbf{e}_{n, k}+\nu \mathbf{e}_{n, k+1}\right)\right| \mathrm{d} \nu \\
& \leq\left(\frac{\omega_{2}-\omega_{1}}{n^{2}}\right)\left(\int_{0}^{1}|\varpi(\nu)|^{p} \mathrm{~d} \nu\right) \sum_{k=0}^{(1 / p)}\left(\int_{0}^{1}\left|f^{\prime}\left((1-v) \mathbf{e}_{n, k}+\nu \mathbf{e}_{n, k+1}\right)\right|^{q} \mathrm{~d} \nu\right)^{(1 / q)} \\
& \leq\left(\frac{\omega_{2}-\omega_{1}}{n^{2}}\right)\left[\frac{2}{p+1}\left(\frac{2^{p+1}+1}{6^{p+1}}\right)\right]^{(1 / p)} \sum_{k=0}^{n-1}\left(\int_{0}^{1}\left[(1-\nu)^{s}\left|f^{\prime}\left(\mathbf{e}_{n, k}\right)\right|^{q}+\nu^{s}\left|f^{\prime}\left(\mathbf{e}_{n, k+1}\right)\right|^{q}\right] \mathrm{d} \nu\right)^{(1 / q)} \\
& =\left(\frac{\omega_{2}-\omega_{1}}{n^{2}}\right)\left[\frac{2}{p+1}\left(\frac{2^{p+1}+1}{6^{p+1}}\right)\right]^{(1 / p)}\left(\frac{1}{s+1}\right)^{(1 / q)} \sum_{k=0}^{n-1}\left[\left|f^{\prime}\left(\mathbf{e}_{n, k}\right)\right|^{q}+\left|f^{\prime}\left(\mathbf{e}_{n, k+1}\right)\right|^{q}\right]^{(1 / q)} .
\end{aligned}
$$


This ends our proof.

Corollary 3. Choosing $s=1$ in Theorem 3, we get the following inequality for the convex function:

$$
\left|T_{n}\left(f ; \omega_{1}, \omega_{2}\right)\right| \leq\left(\frac{\omega_{2}-\omega_{1}}{n^{2} \sqrt{[q]} 2}\right)\left[\frac{2}{p+1}\left(\frac{2^{p+1}+1}{6^{p+1}}\right)\right]^{(1 / p)} \sum_{k=0}^{n-1}\left[\left|f^{\prime}\left(\mathbf{e}_{n, k}\right)\right|^{q}+\left|f^{\prime}\left(\mathbf{e}_{n, k+1}\right)\right|^{q}\right]^{(1 / q)} .
$$

Corollary 4. Choosing $n=1$ in Theorem 3, we get

$$
\begin{aligned}
& \left|\frac{1}{6}\left[f\left(\omega_{1}\right)+4 f\left(\frac{\omega_{1}+\omega_{2}}{2}\right)+f\left(\omega_{2}\right)\right]-\frac{1}{\omega_{2}-\omega_{1}} \int_{\omega_{1}}^{\omega_{2}} f(x) \mathrm{d} x\right| \leq\left(\omega_{2}-\omega_{1}\right) \\
& \quad \times\left[\frac{2}{p+1}\left(\frac{2^{p+1}+1}{6^{p+1}}\right)\right]^{(1 / p)}\left[\frac{\left|f^{\prime}\left(\omega_{1}\right)\right|^{q}+\left|f^{\prime}\left(\omega_{2}\right)\right|^{q}}{s+1}\right]^{(1 / q)} .
\end{aligned}
$$

Theorem 4. Let $f: \mathscr{T} \subseteq[0,+\infty) \longrightarrow \mathbb{R}$ be an $\mathscr{L}^{1}$ function on $\mathscr{T}$ such that $\omega_{1}, \omega_{2} \in \mathscr{T}$ with $\omega_{1}<\omega_{2}$ and $s \in(0,1]$ be fixed.
Also, let $f^{\prime} \in \mathscr{L}^{1}$ and $\left|f^{\prime}\right|^{q}, q \geq 1$ be s-convex function on $\left[\omega_{1}, \omega_{2}\right]$, and then for any positive integer number $n$, we have

$$
\begin{aligned}
\left|T_{n}\left(f ; \omega_{1}, \omega_{2}\right)\right| \leq & \left(\frac{\omega_{2}-\omega_{1}}{n^{2}}\right)\left(\frac{5}{36}\right)^{1-(1 / q)} \\
& \times \sum_{k=0}^{n-1}\left\{\left[A_{1}(s)\left|f^{\prime}\left(\mathbf{e}_{n, k}\right)\right|^{q}+A_{2}(s)\left|f^{\prime}\left(\mathbf{e}_{n, k+1}\right)\right|^{q}\right]^{(1 / q)}+\left[A_{3}(s)\left|f^{\prime}\left(\mathbf{e}_{n, k}\right)\right|^{q}+A_{4}(s)\left|f^{\prime}\left(\mathbf{e}_{n, k+1}\right)\right|^{q}\right]^{(1 / q)}\right\},
\end{aligned}
$$

where $A_{i}(s)$ for all $i=1,2,3,4$ and $\mathbf{e}_{n, k}$ are as defined in Theorem 2.
Proof. By using Lemma 1, the well-known power mean inequality, $s$-convexity of $\left|f^{\prime}\right|^{q}$, and properties of modulus, we have

$$
\begin{aligned}
\left|T_{n}\left(f ; \omega_{1}, \omega_{2}\right)\right| \leq & \left(\frac{\omega_{2}-\omega_{1}}{n^{2}}\right) \sum_{k=0}^{n-1} \int_{0}^{1}|\Phi(\nu)|\left|f^{\prime}\left((1-\nu) \mathbf{e}_{n, k}+\nu \mathbf{e}_{n, k+1}\right)\right| \mathrm{d} \nu \\
\leq & \left(\frac{\omega_{2}-\omega_{1}}{n^{2}}\right)\left(\int_{0}^{1}|\omega(\nu)| \mathrm{d} \nu\right)^{1-(1 / q)} \sum_{k=0}^{n-1}\left(\int_{0}^{1}|\omega(\nu)|\left|f^{\prime}\left((1-v) \mathbf{e}_{n, k}+\nu \mathbf{e}_{n, k+1}\right)\right|^{q} \mathrm{~d} \nu\right)^{(1 / q)} \\
\leq & \left(\frac{\omega_{2}-\omega_{1}}{n^{2}}\right)\left(\frac{5}{36}\right)^{1-(1 / q)} \sum_{k=0}^{n-1}\left(\int_{0}^{1}|\omega(\nu)|\left[(1-\nu)^{s}\left|f^{\prime}\left(\mathbf{e}_{n, k}\right)\right|^{q}+\nu^{s}\left|f^{\prime}\left(\mathbf{e}_{n, k+1}\right)\right|^{q}\right] \mathrm{d} \nu\right)^{(1 / q)} \\
= & \left(\frac{\omega_{2}-\omega_{1}}{n^{2}}\right)\left(\frac{5}{36}\right)^{1-(1 / q)} \\
& \times \sum_{k=0}^{n-1}\left\{\left[A_{1}(s)\left|f^{\prime}\left(\mathbf{e}_{n, k}\right)\right|^{q}+A_{2}(s)\left|f^{\prime}\left(\mathbf{e}_{n, k+1}\right)\right|^{q}\right]^{(1 / q)}+\left[A_{3}(s)\left|f^{\prime}\left(\mathbf{e}_{n, k}\right)\right|^{q}+A_{4}(s)\left|f^{\prime}\left(\mathbf{e}_{n, k+1}\right)\right|^{q}\right]^{(1 / q)}\right\},
\end{aligned}
$$

which completes the proof. 
Corollary 5. Choosing $s=1$ in Theorem 4, we get the following inequality for the convex function:

$$
\begin{aligned}
\left|T_{n}\left(f ; \omega_{1}, \omega_{2}\right)\right| \leq & \left(\frac{\omega_{2}-\omega_{1}}{n^{2}}\right)\left(\frac{5}{36}\right)^{1-(1 / q)} \\
& \times \sum_{k=0}^{n-1}\left\{\left[A_{1}\left|f^{\prime}\left(\mathbf{e}_{n, k}\right)\right|^{q}+A_{2}\left|f^{\prime}\left(\mathbf{e}_{n, k+1}\right)\right|^{q}\right]^{(1 / q)}+\left[A_{3}\left|f^{\prime}\left(\mathbf{e}_{n, k}\right)\right|^{q}+A_{4}\left|f^{\prime}\left(\mathbf{e}_{n, k+1}\right)\right|^{q}\right]^{(1 / q)}\right\},
\end{aligned}
$$

where $A_{i}$ for all $i=1,2,3,4$ are defined as in Corollary 1. Corollary 6. Choosing $n=1$ in Theorem 4, we get

$$
\begin{aligned}
& \left|\frac{1}{6}\left[f\left(\omega_{1}\right)+4 f\left(\frac{\omega_{1}+\omega_{2}}{2}\right)+f\left(\omega_{2}\right)\right]-\frac{1}{\omega_{2}-\omega_{1}} \int_{\omega_{1}}^{\omega_{2}} f(x) \mathrm{d} x\right| \leq\left(\omega_{2}-\omega_{1}\right)\left(\frac{5}{36}\right)^{1-(1 / q)} \\
& \quad \times\left\{\left[A_{1}(s)\left|f^{\prime}\left(\omega_{1}\right)\right|^{q}+A_{2}(s)\left|f^{\prime}\left(\omega_{2}\right)\right|^{q}\right]^{(1 / q)}+\left[A_{3}(s)\left|f^{\prime}\left(\omega_{1}\right)\right|^{q}+A_{4}(s)\left|f^{\prime}\left(\omega_{2}\right)\right|^{q}\right]^{(1 / q)}\right\} .
\end{aligned}
$$

\section{Examples and Applications}

3.1. Bessel Functions. Consider the function $\mathscr{D}_{\tau}:(0, \infty) \longrightarrow$ $[1, \infty)$ given by

$$
\mathscr{D}_{\tau}(x)=2^{\tau} \Gamma(\tau+1) x^{-\tau} \mathscr{M}_{\tau}(x),
$$

where $\mathscr{M}_{\tau}$ is the modified Bessel function of the first kind defined by (see (2) in [46], p. 77):

$$
\mathscr{M}_{\tau}(x)=\sum_{n=0}^{\infty} \frac{(x / 2)^{\tau+2 n}}{n ! \Gamma(\tau+1+n)}, \quad x \in \mathbb{R} .
$$

The first order derivative formula of $\mathscr{D}_{\tau}(x)$ is given by [46]:

$$
\mathscr{D}_{\tau}^{\prime}(x)=\frac{x}{2(\tau+1)} \mathscr{D}_{\tau+1}(x),
$$

and the second derivative can be easily calculated from (27) to be

$$
\mathscr{D}_{\tau}^{\prime \prime}(x)=\frac{x^{2} \mathscr{D}_{\tau+2}(x)}{4(\tau+1)(\tau+2)}+\frac{\mathscr{D}_{\tau+1}(x)}{2(\tau+1)}
$$

Example 1. Let $0<\omega_{1}<\omega_{2}$ and $\tau>-1$. Then, by applying Corollary 2 (note that all assumptions are satisfied) and the identities (27) and (28), we have

$$
\begin{aligned}
& \left|\frac{1}{12(\tau+1)}\left[\omega_{1} \mathscr{D}_{\tau+1}\left(\omega_{1}\right)+\frac{\omega_{1}+\omega_{2}}{2} \mathscr{D}_{\tau+1}\left(\frac{\omega_{1}+\omega_{2}}{2}\right)+\omega_{2} \mathscr{D}_{\tau+1}\left(\omega_{2}\right)\right]-\frac{\mathscr{D}_{\tau}\left(\omega_{2}\right)-\mathscr{D}_{\tau}\left(\omega_{1}\right)}{\omega_{2}-\omega_{1}}\right| \\
& \quad \leq \frac{\omega_{2}-\omega_{1}}{2}\left[\left(A_{1}(s)+A_{3}(s)\right)\left(\frac{\omega_{1}^{2} \mathscr{D}_{\tau+2}\left(\omega_{1}\right)}{2(\tau+1)(\tau+2)}+\frac{\omega_{1} \mathscr{D}_{\tau+1}\left(\omega_{1}\right)}{\tau+1}\right)\right. \\
& \left.\quad+\left(A_{2}(s)+A_{4}(s)\right)\left(\frac{\omega_{2}^{2} \mathscr{D}_{\tau+2}\left(\omega_{2}\right)}{2(\tau+1)(\tau+2)}+\frac{\omega_{2} \mathscr{D}_{\tau+1}\left(\omega_{2}\right)}{\tau+1}\right)\right],
\end{aligned}
$$

where $A_{i}(s), i=1,2,3,4$ are as in Theorem 2 .
Example 2. Let $0<\omega_{1}<\omega_{2}$ and $\tau>-1$. Then, by applying Corollary 4 (note that all assumptions are satisfied) and the identities (27) and (28), we have 


$$
\begin{aligned}
& \left|\frac{1}{12(\tau+1)}\left[\omega_{1} \mathscr{D}_{\tau+1}\left(\omega_{1}\right)+\frac{\omega_{1}+\omega_{2}}{2} \mathscr{D}_{\tau+1}\left(\frac{\omega_{1}+\omega_{2}}{2}\right)+\omega_{2} \mathscr{D}_{\tau+1}\left(\omega_{2}\right)\right]-\frac{\mathscr{D}_{\tau}\left(\omega_{2}\right)-\mathscr{D}_{\tau}\left(\omega_{1}\right)}{\omega_{2}-\omega_{1}}\right| \\
& \leq \frac{\omega_{2}-\omega_{1}}{2(s+1)^{(1 / q)}}\left[\frac{2}{p+1}\left(\frac{2^{p+1}+1}{6^{p+1}}\right)\right]^{(1 / p)}\left[\left(\frac{\omega_{1}^{2} \mathscr{D}_{\tau+2}\left(\omega_{1}\right)}{2(\tau+1)(\tau+2)}+\frac{\omega_{1} \mathscr{D}_{\tau+1}\left(\omega_{1}\right)}{\tau+1}\right)^{q}\right. \\
& \left.\quad+\left(\frac{\omega_{2}^{2} \mathscr{D}_{\tau+2}\left(\omega_{2}\right)}{2(\tau+1)(\tau+2)}+\frac{\omega_{2} \mathscr{D}_{\tau+1}\left(\omega_{2}\right)}{\tau+1}\right)^{q}\right]^{(1 / q)} .
\end{aligned}
$$

Example 3. Let $0<\omega_{1}<\omega_{2}$ and $\tau>-1$. Then, by applying Corollary 6 (note that all assumptions are satisfied) and the identities (27) and (28), we have

$$
\begin{aligned}
& \left|\frac{1}{12(\tau+1)}\left[\omega_{1} \mathscr{D}_{\tau+1}\left(\omega_{1}\right)+\frac{\omega_{1}+\omega_{2}}{2} \mathscr{D}_{\tau+1}\left(\frac{\omega_{1}+\omega_{2}}{2}\right)+\omega_{2} \mathscr{D}_{\tau+1}\left(\omega_{2}\right)\right]-\frac{\mathscr{D}_{\tau}\left(\omega_{2}\right)-\mathscr{D}_{\tau}\left(\omega_{1}\right)}{\omega_{2}-\omega_{1}}\right| \\
& \leq \frac{\omega_{2}-\omega_{1}}{2}\left(\frac{5}{36}\right)^{1-(1 / q)}\left\{\left[A_{1}(s)\left(\frac{\omega_{1}^{2} \mathscr{D}_{\tau+2}\left(\omega_{1}\right)}{2(\tau+1)(\tau+2)}+\frac{\omega_{1} \mathscr{D}_{\tau+1}\left(\omega_{1}\right)}{\tau+1}\right)^{q}\right.\right. \\
& \left.\quad+A_{2}(s)\left(\frac{\omega_{2}^{2} \mathscr{D}_{\tau+2}\left(\omega_{2}\right)}{2(\tau+1)(\tau+2)}+\frac{\omega_{2} \mathscr{D}_{\tau+1}\left(\omega_{2}\right)}{\tau+1}\right)^{q}\right]^{(1 / q)}+\left[A_{3}(s)\left(\frac{\omega_{1}^{2} \mathscr{D}_{\tau+2}\left(\omega_{1}\right)}{2(\tau+1)(\tau+2)}+\frac{\omega_{1} \mathscr{D}_{\tau+1}\left(\omega_{1}\right)}{\tau+1}\right)^{q}\right. \\
& \left.\left.\quad+A_{4}(s)\left(\frac{\omega_{2}^{2} \mathscr{D}_{\tau+2}\left(\omega_{2}\right)}{2(\tau+1)(\tau+2)}+\frac{\omega_{2} \mathscr{D}_{\tau+1}\left(\omega_{2}\right)}{\tau+1}\right)^{q}\right]^{(1 / q)}\right\},
\end{aligned}
$$

where $A_{i}(s), i=1,2,3,4$ are as in Theorem 2 .

3.2. Special Means. We consider the following two special $\mathscr{L}_{r}\left(\omega_{1}, \omega_{2}\right)=\left[\frac{\omega_{2}^{r+1}-\omega_{1}^{r+1}}{(r+1)\left(\omega_{2}-\omega_{1}\right)}\right]^{(1 / r)}, \quad r \in \mathbb{R} \backslash\{-1,0\}$. means for different positive real numbers $\omega_{1}$ and $\omega_{2}$ :

(i) The arithmetic mean:

$$
\mathscr{A}\left(\omega_{1}, \omega_{2}\right)=\frac{\omega_{1}+\omega_{2}}{2}
$$

Proposition 1. Let $n \in \mathbb{N}$ and $0<\omega_{1}<\omega_{2}$. Then, we have for a fixed $s \in(0,1]$

(ii) The generalized logarithmic mean:

$$
\begin{aligned}
& \mid \sum_{k=0}^{n-1} \frac{1}{3 n}\left[\mathscr{A}\left(\left(\frac{(n-k) \omega_{1}+k \omega_{2}}{n}\right)^{s},\left(\frac{(n-k-1) \omega_{1}+(k+1) \omega_{2}}{n}\right)^{s}\right)\right. \\
& \left.\quad+\frac{2}{n^{s}} \mathscr{A}\left((2(n-k)-1) \omega_{1},(2 k+1) \omega_{2}\right)\right]-\mathscr{L}_{s}^{s}\left(\omega_{1}, \omega_{2}\right) \mid \leq \frac{2^{s-1} s}{n^{s+1}}\left(\omega_{2}-\omega_{1}\right) \\
& \quad \times \sum_{k=0}^{n-1}\left[\left(A_{1}(s)+A_{3}(s)\right) \mathscr{A}^{s-1}\left((n-k) \omega_{1}, k \omega_{2}\right)+\left(A_{2}(s)+A_{4}(s)\right) \mathscr{A}^{s-1}\left((n-k-1) \omega_{1},(k+1) \omega_{2}\right)\right],
\end{aligned}
$$

where $A_{i}(s)$ for all $i=1,2,3,4$ are as defined in Theorem 2.

Proof. By applying Theorem 2 with $f(x)=x^{s}, x \in\left[\omega_{1}, \omega_{2}\right]$ and $s \in(0,1]$, then we can obtain the desired result (34).
Proposition 2. Let $n \in \mathbb{N}$ and $0<\omega_{1}<\omega_{2}$. Then, we have for a fixed $s \in(0,1]$ 


$$
\begin{aligned}
\mid \sum_{k=0}^{n-1} & \frac{1}{3 n}\left[\mathscr{A}\left(\left(\frac{(n-k) \omega_{1}+k \omega_{2}}{n}\right)^{s},\left(\frac{(n-k-1) \omega_{1}+(k+1) \omega_{2}}{n}\right)\right)\right. \\
& \left.+\frac{2}{n^{s}} \mathscr{A}\left((2(n-k)-1) \omega_{1},(2 k+1) \omega_{2}\right)\right]-\mathscr{L}_{s}^{s}\left(\omega_{1}, \omega_{2}\right) \mid \\
\leq & \frac{2^{s-1} s}{n^{s+1}}\left(\omega_{2}-\omega_{1}\right)\left[\frac{2}{p+1}\left(\frac{2^{p+1}+1}{6^{p+1}}\right)\right]^{(1 / p)}\left(\frac{1}{s+1}\right)^{(1 / q)} \\
& \times \sum_{k=0}^{n-1}\left[\mathscr{A}^{q(s-1)}\left((n-k) \omega_{1}, k \omega_{2}\right)+\mathscr{A}^{q(s-1)}\left((n-k-1) \omega_{1},(k+1) \omega_{2}\right)\right]^{(1 / q)},
\end{aligned}
$$

where $(1 / p)+(1 / q)=1$ with $q>1$.

Proof. By applying Theorem 3 with $f(x)=x^{s}, x \in\left[\omega_{1}, \omega_{2}\right]$ and $s \in(0,1]$, we can obtain the desired result (37) directly.
Proposition 3. Let $n \in \mathbb{N}$ and $0<\omega_{1}<\omega_{2}$. Then, we have for a fixed $s \in(0,1]$

$$
\begin{aligned}
\mid & \sum_{k=0}^{n-1} \frac{1}{3 n}\left[\mathscr{A}\left(\left(\frac{(n-k) \omega_{1}+k \omega_{2}}{n}\right)^{s},\left(\frac{(n-k-1) \omega_{1}+(k+1) \omega_{2}}{n}\right)^{s}\right)\right. \\
& \left.+\frac{2}{n^{s}} \mathscr{A}\left((2(n-k)-1) \omega_{1},(2 k+1) \omega_{2}\right)\right]-\mathscr{L}_{s}^{s}\left(\omega_{1}, \omega_{2}\right) \mid \leq \frac{2^{s-1} s}{n^{s+1}}\left(\omega_{2}-\omega_{1}\right)\left(\frac{5}{36}\right)^{1-(1 / q)} \\
& \times \sum_{k=0}^{n-1}\left\{\left[A_{1}(s) \mathscr{A}^{q(s-1)}\left((n-k) \omega_{1}, k \omega_{2}\right)+A_{2}(s) \mathscr{A}^{q(s-1)}\left((n-k-1) \omega_{1},(k+1) \omega_{2}\right)\right]^{(1 / q)}\right. \\
& \left.+\left[A_{3}(s) \mathscr{A}^{q(s-1)}\left((n-k) \omega_{1}, k \omega_{2}\right)+A_{4}(s) \mathscr{A}^{q(s-1)}\left((n-k-1) \omega_{1},(k+1) \omega_{2}\right)\right]^{(1 / q)}\right\}
\end{aligned}
$$

where $A_{i}(s)$ for all $i=1,2,3,4$ are defined as in Theorem 2 and $q \geq 1$.

Proof. By applying Theorem 4 with $f(x)=x^{s}, x \in\left[\omega_{1}, \omega_{2}\right]$ and $s \in(0,1]$, we can directly obtain the desired result (43).

3.3. Simpson Quadrature Formula. Let $\mathscr{P}$ be the partition of the points $\omega_{1}=x_{0}<x_{1}<\cdots<x_{l}=\omega_{2}$ of the interval $\left[\omega_{1}, \omega_{2}\right]$ and consider the quadrature formula

$$
\int_{\omega_{1}}^{\omega_{2}} f(x) \mathrm{d} x=\mathcal{S}_{n}^{(i)}(f, \mathscr{P})+\mathscr{E}(f, \mathscr{P}),
$$

where

$$
\begin{aligned}
\mathcal{S}_{n}^{(i)}(f, \mathscr{P}):= & \sum_{k=0}^{n-1} \frac{1}{6 n}\left[f\left(\frac{(n-k) x_{i}+k x_{i+1}}{n}\right)\right. \\
& +4 f\left(\frac{(2(n-k)-1) x_{i}+(2 k+1) x_{i+1}}{2 n}\right) \\
& \left.+f\left(\frac{(n-k-1) x_{i}+(k+1) x_{i+1}}{n}\right)\right]\left(x_{i+1}-x_{i}\right)
\end{aligned}
$$

is the Simpson version and $\mathscr{E}(f, \mathscr{P})$ denotes the associated approximation error. In this section, we are going to derive some new estimates for the Simpson quadrature formula.

Proposition 4. Let $0<\omega_{1}<\omega_{2}, s \in(0,1]$ be fixed and $f:\left[\omega_{1}, \omega_{2}\right] \longrightarrow \mathbb{R}$ be a differentiable function on $\left(\omega_{1}, \omega_{2}\right)$. If $f^{\prime} \in \mathscr{L}^{1}\left[\omega_{1}, \omega_{2}\right]$ and $\left|f^{\prime}\right|$ is an s-convex function on $\left[\omega_{1}, \omega_{2}\right]$, then we have

$$
\begin{aligned}
|\mathscr{E}(f, \mathscr{P})| \leq & \sum_{i=0}^{l-1}\left(\frac{x_{i+1}-x_{i}}{n}\right)^{2} \sum_{k=0}^{n-1}\left[\left(A_{1}(s)+A_{3}(s)\right)\left|f^{\prime}\left(\mathbf{e}_{n, k}^{(i)}\right)\right|\right. \\
& \left.+\left(A_{2}(s)+A_{4}(s)\right)\left|f\left(\mathbf{e}_{n, k+1}^{(i)}\right)\right|\right],
\end{aligned}
$$

where $A_{i}(s)$ for all $i=1,2,3,4$ are as defined in Theorem 2 and

$$
\mathbf{e}_{n, j}^{(i)}:=\frac{(n-j) x_{i}+j x_{i+1}}{n}, \quad \text { for } j=k, k+1
$$

Proof. By applying Theorem 2 on the subintervals $\left[x_{i}, x_{i+1}\right](i=0, \ldots, l-1)$ of the partition $\mathscr{P}$, we can deduce 


$$
\begin{aligned}
& \mid \int_{x_{i}}^{x_{i+1}} f(x) d x-\sum_{k=0}^{n-1} \frac{1}{6 n}\left[f\left(\frac{(n-k) x_{i}+k x_{i+1}}{n}\right)+4 f\left(\frac{(2(n-k)-1) x_{i}+(2 k+1) x_{i+1}}{2 n}\right)\right. \\
& \left.\quad+f\left(\frac{(n-k-1) x_{i}+(k+1) x_{i+1}}{n}\right)\right]\left(x_{i+1}-x_{i}\right) \mid \\
& \quad \leq\left(\frac{x_{i+1}-x_{i}}{n}\right)^{2} \sum_{k=0}^{n-1}\left[\left(A_{1}(s)+A_{3}(s)\right)\left|f^{\prime}\left(\mathbf{e}_{n, k}^{(i)}\right)\right|+\left(A_{2}(s)+A_{4}(s)\right)\left|f^{\prime}\left(\mathbf{e}_{n, k+1}^{(i)}\right)\right|\right] .
\end{aligned}
$$

Therefore, from (41), it follows that

$$
\begin{aligned}
|\mathscr{E}(f, \mathscr{P})|= & \left|\int_{\omega_{1}}^{\omega_{2}} f(x) \mathrm{d} x-\mathcal{S}_{n}^{(i)}(f, \mathscr{P})\right| \leq \sum_{i=0}^{l-1} \mid \int_{x_{i}}^{x_{i+1}} f(x) \mathrm{d} x-\sum_{k=0}^{n-1} \frac{1}{6 n}\left[f\left(\frac{(n-k) x_{i}+k x_{i+1}}{n}\right)\right. \\
& \left.+4 f\left(\frac{(2(n-k)-1) x_{i}+(2 k+1) x_{i+1}}{2 n}\right)+f\left(\frac{(n-k-1) x_{i}+(k+1) x_{i+1}}{n}\right)\right]\left(x_{i+1}-x_{i}\right) \mid \\
\leq & \sum_{i=0}^{l-1}\left(\frac{x_{i+1}-x_{i}}{n}\right)^{2} \sum_{k=0}^{n-1}\left[\left(A_{1}(s)+A_{3}(s)\right)\left|f^{\prime}\left(\mathbf{e}_{n, k}^{(i)}\right)\right|+\left(A_{2}(s)+A_{4}(s)\right)\left|f^{\prime}\left(\mathbf{e}_{n, k+1}^{(i)}\right)\right|\right] .
\end{aligned}
$$

This gives our Proposition 4.

Proposition 5. Let $0<\omega_{1}<\omega_{2}, s \in(0,1]$ be fixed and $f:\left[\omega_{1}, \omega_{2}\right] \longrightarrow \mathbb{R}$ be a differentiable function on $\left(\omega_{1}, \omega_{2}\right)$. If

$f^{\prime} \in \mathscr{L}^{1}\left[\omega_{1}, \omega_{2}\right]$ and $\left|f^{\prime}\right|^{q}$ is an s-convex function on $\left[\omega_{1}, \omega_{2}\right]$, then we have for $q>1$ and $(1 / p)+(1 / q)=1$ :

$$
|\mathscr{E}(f, \mathscr{P})| \leq\left[\frac{2}{p+1}\left(\frac{2^{p+1}+1}{6^{p+1}}\right)\right]^{(1 / p)}\left(\frac{1}{s+1}\right)^{(1 / q)} \sum_{i=0}^{l-1}\left(\frac{x_{i+1}-x_{i}}{n}\right)^{2} \sum_{k=0}^{n-1}\left[\left|f^{\prime}\left(\mathbf{e}_{n, k}^{(i)}\right)\right|^{q}+\left|f^{\prime}\left(\mathbf{e}_{n, k+1}^{(i)}\right)\right|^{q}\right]^{(1 / q)}
$$

where $\mathbf{e}_{n, k}^{(i)}$ and $\mathbf{e}_{n, k+1}^{(i)}$ are as defined in Proposition 4.

Proof. By the same method used for Proposition 4 and by using Theorem 3, one can prove this proposition directly.
Proposition 6. Let $0<\omega_{1}<\omega_{2}, s \in(0,1]$ be fixed and $f:\left[\omega_{1}, \omega_{2}\right] \longrightarrow \mathbb{R}$ be a differentiable function on $\left(\omega_{1}, \omega_{2}\right)$. If $f^{\prime} \in \mathscr{L}^{1}\left[\omega_{1}, \omega_{2}\right]$ and $\left|f^{\prime}\right|^{q}$ is an s-convex function on $\left[\omega_{1}, \omega_{2}\right]$, then we have for $q \geq 1$ :

$$
\begin{aligned}
|\mathscr{E}(f, \mathscr{P})| \leq & \left(\frac{5}{36}\right)^{1-(1 / q)} \sum_{i=0}^{l-1}\left(\frac{x_{i+1}-x_{i}}{n}\right)^{2} \sum_{k=0}^{n-1}\left\{\left[A_{1}(s)\left|f^{\prime}\left(\mathbf{e}_{n, k}^{(i)}\right)\right|^{q}+A_{2}(s)\left|f^{\prime}\left(\mathbf{e}_{n, k+1}^{(i)}\right)\right|^{q}\right]^{(1 / q)}\right. \\
& \left.+\left[A_{3}(s)\left|f^{\prime}\left(\mathbf{e}_{n, k}^{(i)}\right)\right|^{q}+A_{4}(s)\left|f^{\prime}\left(\mathbf{e}_{n, k+1}^{(i)}\right)\right|^{q}\right]^{(1 / q)}\right\},
\end{aligned}
$$

where $\mathbf{e}_{n, k}^{(i)}$ and $\mathbf{e}_{n, k+1}^{(i)}$ are as defined in Proposition 4.

Proof. By the same method used for Proposition 4 and by using Theorem 4, one can prove this proposition directly.

\section{Conclusion}

We have obtained a new Simpson identity at first. By using this, we have established some interesting Simpson's type integral inequalities for $s$-convex functions. In order to illustrate the efficiency of our main results, some applications to the Bessel functions, special means, and error estimation 
about Simpson quadrature formula are provided. To the best of our knowledge, these results are new in the literature. Since the class of convex ( $s$-convex) functions have large applications in many mathematical areas, they can be applied to obtain several results in convex analysis, quantum mechanics, special functions, mathematical inequalities, and related optimization theory and may stimulate further research in different areas of pure and applied sciences.

\section{Data Availability}

No data were used to support this study.

\section{Conflicts of Interest}

The authors declare that they have no conflicts of interest.

\section{Authors' Contributions}

All authors contributed equally and significantly in writing this article. All authors read and approved the final manuscript.

\section{Acknowledgments}

The third author would like to thank Prince Sultan University for funding this work through the research group Nonlinear Analysis Methods in Applied Mathematics (NAMAM) (group number RG-DES-2017-01-17).

\section{References}

[1] M. J. Cloud, B. C. Drachman, and L. Lebedev, Inequalities, Springer, Cham, Switzerland, Second edition, 2014.

[2] Y. El-Khatib and Q. M. Al-Mdallal, "Numerical simulations for the pricing of options in jump diffusion markets," Arab Journal of Mathematical Sciences, vol. 18, no. 2, pp. 199-208, 2012.

[3] L. Al Sakkaf, Q. Al-Mdallal, and U. Al Khawaja, "A numerical algorithm for solving higher-order nonlinear BVPs with an application on fluid flow over a shrinking permeable infinite long cylinder," Complexity, vol. 2018, Article ID 8269541, 11 pages, 2018.

[4] F. Haq, K. Shah, Q. M. Al-Mdallal, and F. Jarad, "Application of a hybrid method for systems of fractional order partial differential equations arising in the model of the one-dimensional Keller-Segel equation," The European Physical Journal Plus, vol. 134, no. 9, p. 461, 2019.

[5] Q. Al-Mdallal, M. Syam, M. D. K. Al-Srihin, and M. Al-Refai, "Theoretical and computational perspectives on the eigenvalues of fourth-order fractional Sturm-Liouville problem," International Journal of Computer Mathematics, vol. 95, no. 8, pp. 1548-1564, 2018.

[6] U. Al Khawaja and Q. M. Al-Mdallal, "Convergent power series of and solutions to nonlinear differential equations," International Journal of Differential Equations, vol. 2018, Article ID 6043936, 2018.

[7] A. A. Kilbas, H. M. Srivastava, and J. J. Trujillo, "Theory and applications of fractional differential equations," North-Holland Mathematics Studies, Vol. 204, Elsevier Science B.V., Amsterdam, The Netherlands, 1st edition, 2006.

[8] D. Baleanu, P. O. Mohammed, M. Vivas-Cortez, and Y. Rangel-Oliveros, "Some modifications in conformable fractional integral inequalities," Advances in Difference Equations, vol. 2020, p. 374, 2020.

[9] S. S. Dragomir and R. P. Agarwal, "Two inequalities for differentiable mappings and applications to special means of real numbers and to trapezoidal formula," Applied Mathematics Letters, vol. 11, no. 5, pp. 91-95, 1998.

[10] C. E. M. Pearce and J. Pečarić, "Inequalities for differentiable mappings with application to special means and quadrature formulæ," Applied Mathematics Letters, vol. 13, no. 2, pp. 51-55, 2000.

[11] U. S. Kirmaci, "Inequalities for differentiable mappings and applications to special means of real numbers and to midpoint formula," Applied Mathematics and Computation, vol. 147, no. 1, pp. 137-146, 2004.

[12] F. M. Atıc1 and H. Yaldı, "Convex functions on discrete time domains," Canadian Mathematical Bulletin, vol. 59, pp. 225-233, 2016.

[13] H. Yaldiz and P. Agarwal, "s-convex functions on discrete time domains," Analysis, vol. 37, pp. 179-184, 2017.

[14] P. O. Mohammed and M. Z. Sarikaya, "On generalized fractional integral inequalities for twice differentiable convex functions," Journal of Computational and Applied Mathematics, vol. 372, Article ID 112740, 2020.

[15] I. Gavrea, "On some inequalities for convex functions," Journal of Mathematical Inequalities, vol. 3, no. 3, pp. 315-321, 2009.

[16] T.-Y. Zhang, A.-P. Ji, and F. Qi, "On integral inequalities of Hermite-Hadamard type for s-geometrically convex functions," Abstract and Applied Analysis, vol. 2012, Article ID 560586, 2012.

[17] T.-Y. Zhang, A.-P. Ji, and F. Qi, "Some inequalities of Hermite-Hadamard type for GA-convex functions with applications to means," Le Matematiche, vol. 68, pp. 229-239, 2013.

[18] P. O. Mohammed, M. Vivas-Cortez, T. Abdeljawad, and Y. Rangel-Oliveros, "Integral inequalities of Hermite-Hadamard type for quasi-convex functions with applications," AIMS Mathematics, vol. 5, pp. 7316-7331, 2020.

[19] P. O. Mohammed, "Some new Hermite-Hadamard type inequalities for MT-convex functions on differentiable coordinates," Journal of King Saud University-Science, vol. 30, no. 2, pp. 258-262, 2018.

[20] J. Han, P. O. Mohammed, and H. Zeng, "Generalized fractional integral inequalities of Hermite-Hadamard-type for a convex function," Open Mathematics, vol. 18, no. 1, pp. 794-806, 2020.

[21] D.-P. Shi, B.-Y. Xi, and F. Qi, "Hermite-Hadamard type inequalities for Riemann-Liouville fractional integrals of $(\alpha, m)$-convex functions," Fractional Differential Calculus, vol. 4, no. 1, pp. 31-43, 2014.

[22] P. O. Mohammed and M. Z. Sarikaya, "Hermite-Hadamard type inequalities for $F$-convex function involving fractional integrals," Journal of Inequalities and Applications, vol. 2018, p. 359, 2018.

[23] D. Baleanu, P. O. Mohammed, and S. Zeng, "Inequalities of trapezoidal type involving generalized fractional integrals," Alexandria Engineering Journal, vol. 59, no. 5, pp. 2975-2984, 2020.

[24] P. O. Mohammed, T. Abdeljawad, S. Zeng, and A. Kashuri, "Fractional Hermite-Hadamard integral inequalities for a new class of convex functions," Symmetry, vol. 12, no. 9, p. 1485, 2020.

[25] S. S. Dragomir and C. E. M. Pearce, Selected Topics on Hermite-Hadamard Inequalities and Applications; RGMIA Monographs, Victoria University, Footscray, Australia, 2000. 
[26] H. Hudzik and L. Maligranda, "Some remarks ons-convex functions," Aequationes Mathematicae, vol. 48, no. 1, pp. 100-111, 1994.

[27] P. O. Mohammed and I. Brevik, "A new version of the Hermite-Hadamard inequality for Riemann-Liouville fractional integrals," Symmetry, vol. 12, no. 4, p. 610, 2020.

[28] A. Fernandez and P. Mohammed, "Hermite-Hadamard inequalities in fractional calculus defined using Mittag-Leffler kernels," Mathematical Methods in the Applied Sciences, pp. 1-18, 2020.

[29] P. O. Mohammed, T. Abdeljawad, and A. Kashuri, "Fractional Hermite-Hadamard-Fejer inequalities for a convex function with respect to an increasing function involving a positive weighted symmetric function," Symmetry, vol. 12, no. 9, p. $1503,2020$.

[30] B. Gavrea and I. Gavrea, "On some Ostrowski type inequalities," General Mathematics, vol. 18, pp. 33-44, 2010.

[31] S. Kaijser, L. Nikolova, L.-E. Persson, and A. Wedestig, "Hardy-type inequalities via convexity," Mathematical Inequalities \& Applications, vol. 8, no. 3, pp. 403-417, 2005.

[32] H. Gunawan and E. Eridani, "Fractional integrals and generalized Olsen inequalities," Kyungpook Mathematical Journal, vol. 49, no. 1, pp. 31-39, 2009.

[33] Y. Sawano and H. Wadade, "On the Gagliardo-Nirenberg type inequality in the critical Sobolev-Morrey space," Journal of Fourier Analysis and Applications, vol. 19, no. 1, pp. 20-47, 2013.

[34] P. O. Mohammed and T. Abdeljawad, "Opial integral inequalities for generalized fractional operators with nonsingular kernel," Journal of Inequalities and Applications, vol. 2020, p. 148, 2020.

[35] M. Z. Sarikaya, C. C. Bilisik, and P. O. Mohammed, "Some generalizations of Opial type inequalities," Applied Mathematics \& Information Sciences, vol. 14, pp. 809-816, 2020.

[36] C.-J. Zhao and W.-S. Cheung, "On improvements of the Rozanova's inequality," Journal of Inequalities and Applications, vol. 2011, p. 33, 2011.

[37] S. S. Dragomir, R. P. Agarwal, and P. Cerone, "On Simpson's inequality and applications," Journal of Inequalities and Applications, vol. 5, no. 6, pp. 533-579, 2000.

[38] M. Vivas-Cortez, T. Abdeljawad, P. O. Mohammed, and Y. Rangel-Oliveros, "Simpson's integral inequalities for twice differentiable convex functions," Mathematical Problems in Engineering, vol. 2020, Article ID 1936461, 2020.

[39] T. Abdeljawad, S. Rashid, Z. Hammouch, and Y.-M. Chu, "Some new local fractional inequalities associated with generalized $(s, m)$-convex functions and applications," Advances in Difference Equations, vol. 2020, p. 406, 2020.

[40] M. U. Awan, S. Talib, A. Kashuri, M. A. Noor, and Y.-M. Chu, "Estimates of quantum bounds pertaining to new q-integral identity with applications," Advances in Difference Equations, vol. 2020, p. 424, 2020.

[41] C.-Y. Luo, T.-S. Du, M. Kunt, and Y. Zhang, "New bounds considering the weighted Simpson-like type inequality and applications," Journal of Inequalities and Applications, vol. 2018, p. 332, 2018.

[42] J. Pečarić and S. Varošanec, “A note on Simpson's inequality for functions of bounded variation," Tamkang Journal of Mathematics, vol. 31, pp. 239-242, 2000.

[43] S. Qaisar, C. He, and S. Hussain, "A generalizations of Simpson's type inequality for differentiable functions using $(\alpha, m)$-convex functions and applications," Journal of Inequalities and Applications, vol. 2013, p. 158, 2013.
[44] M. Z. Sarikaya, E. Set, and E. Özdemir, "On new inequalities of Simpson's type for s-convex functions," Computers \& Mathematics with Applications, vol. 60, no. 8, pp. 2191-2199, 2010.

[45] N. Ujević, "Sharp inequalities of Simpson type and Ostrowski type," Computers \& Mathematics with Applications, vol. 48, no. 1-2, pp. 145-151, 2004.

[46] G. N. Watson, A Treatise on the Theory of Bessel Functions, Cambridge Mathematical Library, Cambridge University Press, Cambridge, England, 1995. 